\title{
Multivariable Methods Applied to FTIR: A Powerful Technique to Highlight Architectural Changes in Poly(lactic Acid)
}

\author{
J.R. Riba ${ }^{*}$, J. Cailloux ${ }^{2}$, R. Cantero ${ }^{1}$, T.Canals ${ }^{1}$, M. Ll. Maspoch ${ }^{2}$ \\ ${ }^{1}$ Escola d'Enginyeria d'Igualada - Universitat Politecnica de Catalunya Barcelona Tech \\ (EEI-UPC), Av. Pla de la Massa, 8, Igualada 08700, Spain \\ ${ }^{2}$ Centre Català del Plàstic (CCP) - Universitat Politecnica de Catalunya Barcelona Tech \\ (EEBE-UPC), C/Colom, 114, Terrassa 08222, Spain \\ * Corresponding author: jordi.riba@eei.upc.edu
}

\begin{abstract}
The structural modifications of a commercially available poly(lactid acid) grade were induced through reactive extrusion using a multi-epoxide reactive agent in a pilot plant. The statistical nature of the chemical reactions led to the generation of several types of nonuniform molecular architectures. Even though conventional spectroscopic (NMR) or chromatographic (SEC-static light scattering) techniques are placed at the forefront of the molecular characterization, both methods usually failed in characterizing non-uniform structures. In this study, a promising approach was applied to automatically classify modified PLA samples. It is based on the analysis of FTIR spectral data by means of multivariable methods, including feature extraction and classification algorithms. The fast and accurate results presented in this paper show the potential of the proposed approach.
\end{abstract}

Keywords: Infrared spectroscopy, Multivariable methods, Process control, Poly(lactic acid), Reactive extrusion 


\section{Introduction}

Among biodegradable polymers, poly(lactic acid) (PLA) has been viewed as a promising alternative to petroleum-based commodity polymers (PET, PS, etc.). Nevertheless, the use of PLA in some specific processing techniques (i.e. sheet casting extrusion, blown film, foaming) is complicated. This is due to its limited melt strength and elasticity, as well as its insufficient thermal stability at high temperatures. On this basis, reactive extrusion (referred to as REx), combined with a multifunctional reactive agent, has been considered as a promising method to both stabilize and enhance PLA melt properties [1,2]. Among the most commonly used chain extenders, the different reactive species able to react with PLA functional end groups (i.e. $-\mathrm{COOH}$ and $-\mathrm{OH}$ ) are epoxy [3], isocyanate [4], anhydride [5] and isocyanurate $[5,6]$ functions.

Until today, a styrene-acrylic multifunctional epoxy random oligomer (SAmfE) has been reported to be the most effective reactive agent to enhance both the melt strength and the thermal stability of PLA during processing [3,7]. This is due to the possible chemical bonding of several PLA chains to one SAmfE molecule, thus leading to the formation of branched macromolecules, which potentially hold long chain branching (LCB). However, due to the statistical nature of the chemical bonding coupled with a possible competition between chain degradation, chain extension, and chain branching, the topological modifications induced in the initial PLA matrix are not trivial. It would be a mixture of several types of nonuniform structures in molecular weight (MW), in architectures and in the number of branch per macromolecules

Conventional spectroscopic (i.e. NMR) or chromatographic (i.e. SEC-static light scattering, SEC-LS) techniques are usually placed at the forefront of the molecular characterization. Unfortunately, both methods are likely to exhibit a limited sensitivity for non-uniform LCB PLA macromolecules, as already reported elsewhere [7]. While the former cannot distinguish branches of six carbon atoms length and longer, as largely reported for polyolefins [8], unreliable LS data are expected using the latter due to the extremely small refractive index increment value $\left(\mathrm{dn} / \mathrm{dc} \approx 0.024 \mathrm{~mL} . \mathrm{g}^{-1}\right.$ in chloroform [9]) exhibited by PLA. Fourier Transform Infrared spectroscopy (FTIR) is another method to evidence possible coupling reactions between PLA functional end groups and the epoxy groups of the SAmfE. Nevertheless, unreliable results are usually obtained due to a content of new covalent bonding, possibly below the detection limits of this technique. That is, the identification of possible subtle differences between raw FTIR data is not trivial.

As the literature reports, multivariable mathematical methods applied to FTIR is a promising technique to identify slight differences between spectra. The computing analysis of principal components (PCA) together with canonical variables (CVA) has already been successfully used in food authenticity testing, the rubber industry and paper identification, among others [10-14]. FTIR is a non-destructive technique, which makes it a suitable tool for process control applications in industrial settings. Accordingly, this works aims at evaluating the applicability of the PCA-CVA multivariable methods coupled to FTIR to highlight structural changes in PLA polymer modified through reactive extrusion in a pilot plant. 


\section{Experimental}

\subsection{Materials}

A commercial PLA extrusion grade (Ingeo 4032D ${ }^{\circledR}$ ), purchased from NatureWorks LLC was used in this study. It has a melt flow index $\left(210^{\circ} \mathrm{C} / 2.16 \mathrm{~kg}\right)$ of $6.4 \pm 0.3 \mathrm{~g} / 10 \mathrm{~min}$ and a Dlactide content of about $2 \%$. SAmfE reactive agent, namely Joncryl-ADR-4300F®, was kindly supplied by BASF in flake form with an epoxy equivalent weight of $433 \mathrm{~g} \cdot \mathrm{mol}^{-1}$ and features a functionality $\sim 12$.

\subsection{Reactive-extrusion calendering}

Prior to processing, raw PLA pellets (referred to as $\mathrm{PLA}_{\text {pellets}}$ ) were dried for $3 \mathrm{~h}$ at $80^{\circ} \mathrm{C}$ in a PIOVAN (DSN506HE, Venice, Italy) hopper-dryer (dew point of $-40{ }^{\circ} \mathrm{C}$ ) and kept during the same conditions during the whole process. SAmfE was powdered using mortar and pestle and then stored overnight at room temperature under vacuum. During all processing stages, a $\mathrm{N}_{2}$ blanket was established in the feeding zone while vacuum was applied in the metering zone of the intermeshing co-rotating twin-screw extruder COLLIN Kneter 25X24D (COLLIN GmbH, Ebersberg, Germany), which features a screw diameter of $25 \mathrm{~mm}$ $(\mathrm{L} / \mathrm{D}=36)$.

Initially, the modification of the PLA molecular structure was achieved through reactive extrusion using a nominal SAmfE content of $0.6 \mathrm{wt} . \%$. The seven heating zones were set to $45,165,165,170,180,190$ and $190^{\circ} \mathrm{C}$ from the feeding zone to the die, respectively. The residence time $\left(\mathrm{t}_{\text {res }}\right)$ was maximized using a screw speed of $35 \mathrm{rpm}$; thus leading to a maximum $t_{\text {res }}$ of $4.1 \mathrm{~min}$. The extrudate (referred to as PLA $A_{\text {REx-pellets }}$ ) was water-cooled, pelletized and crystallized in a convection oven at $90^{\circ} \mathrm{C}$ for $4 \mathrm{~h}$.

In a second step, $15 \mathrm{~m}$ of calendered sheets (nominal width of $100 \mathrm{~mm}$ and nominal thickness of $0.6 \mathrm{~mm}$ ) of pre-dried PLA $A_{\text {REx-pellets }}$ were calendered (referred to as PLA $A_{\text {REx-sheet }}$ ). The screw speed was set to $60 \mathrm{rpm}$ and the seven heating zones were set to 150, 170, 180, 190, 195, 200 and $200^{\circ} \mathrm{C}$ from the feeding zone to the die, respectively. The chill roll temperature was set to $50^{\circ} \mathrm{C}$. For the sake of comparison, a calendered sheet of PLA (referred to as PLA sheet $_{\text {) was }}$ extruded using the same thermo-mechanical history. All prepared samples are summarized in Table 1.

Table 1 Nomenclature of all investigated samples.

\begin{tabular}{lccc}
\hline Sample code & $\begin{array}{c}\mathbf{M}_{\mathbf{n}}{ }^{\mathbf{a}} \\
\left(\text { g.mol }^{-\mathbf{1}}\right)\end{array}$ & $\begin{array}{c}\mathbf{M}_{\mathbf{w}}{ }^{\mathbf{a}} \\
\left(\mathbf{g . m o l}^{-\mathbf{1}}\right)\end{array}$ & $\begin{array}{c}\text { Number of sample } \\
\text { analysed }\end{array}$ \\
\hline PLA $_{\text {pellets }}$ & $90 \pm 1$ & $181 \pm 1$ & 22 \\
PLA $_{\text {sheet }}$ & $85 \pm 1$ & $179 \pm 1$ & 21 \\
PLA $_{\text {REx-pellets }}$ & $110 \pm 1$ & $280 \pm 2$ & 20 \\
PLA $_{\text {REx }}$ & $120 \pm 1$ & $292 \pm 1$ & 16 \\
\hline ght-average molecular weight values determined using SEC-DRI experiments.
\end{tabular}

a Relative weight-average molecular weight values determined using SEC-DRI experiments. 


\subsection{Molecular weight characterization}

The number- and weight-average MW $\left(\mathrm{M}_{\mathrm{n}}\right.$ and $\mathrm{M}_{\mathrm{w}}$, respectively) were determined from sizeexclusion chromatography (SEC) analysis using an Agilent HPLC model Infinity 1260 SEC system (Agilent Technologies, Santa Clara, CA, USA) equipped with a differential refractive detector (SEC-DRI) at room temperature. An Agilent PL hexafluoroisopropanol (HFIP) gel specialist column was used at a flow rate of $2 \mathrm{~mL} \cdot \mathrm{min}^{-1}$. HFIP, containing $0.02 \mathrm{~mol} \cdot \mathrm{L}^{-1}$ of sodium trifluoroisopropanol to prevent polyelectrolyte effect was used as eluent. $M_{n}$ and $M_{w}$ were calculated by reference to a relative calibration curve made of poly(methyl methacrylate) standards.

\subsection{Infrared spectroscopy}

Reflectance spectra were acquired at $25 \pm 1^{\circ} \mathrm{C}$, using a Spectrum One (S/N 57458) FTIR spectrometer from PerkinElmer (Beaconsfield, UK) equipped with an ATR module (Universal Sampling Accessory, S/N P0DL01101418). Spectra were obtained within the wavenumber interval ranging from $4000-650 \mathrm{~cm}^{-1}$, with a total of 4 scans per sample and a resolution of $1 \mathrm{~cm}^{-1}$.

\section{Multivariate data analysis methods}

Under the experimental conditions used in the current study, FTIR analysis provides a large amount of spectral data (i.e. 3351 points per spectrum). Consequently, it is necessary to use fast and efficient multivariate mathematical methods to drive this technique industrially profitable. In a first step, this mathematical analysis aims at condensing the analytically relevant data into a reduced set of inferred or latent variables $[13,15]$. The latent variables are calculated by applying weighted combinations of the original set of variables. Besides the significant data reduction, these methods (named feature extraction methods) are also useful to remove experimental noise exhibited by raw data. Feature extraction methods can be broadly classified into unsupervised and supervised methods. Regarding classification purposes, the latter often provide more accurate results than the former, as they use class tags to assess the behavior of the latent variable. Discrimination among classes is enhanced by using supervised feature extraction algorithms, since an expert user guides the process by selecting the class tags of the samples in the calibration set [13].

Canonical variate analysis (referred to as CVA) is a multi-class supervised feature extraction method. It has been specially designed to enhance differences between different data groups which are under consideration [16]. CVA analyzes samples in the space defined by the original variables (reflectance for each wavenumber in the analyzed problem) and finds the axes or directions in the space strengthening the differences among the different data groups [17]. This process is carried out by maximizing the distance among the different classes defined in the problem, and simultaneously minimizing the distance within classes. CVA produces non-orthogonal latent variables, that is, the canonical variates (referred to as CVs). CVA calculates as many CVs as the number of classes defined in the problem minus one. However, the main drawback of this algorithm is that it requires data sets with a number of samples greater than the number of original variables in the problem. When dealing with 
FTIR spectral data featuring 3351 variables, this condition requires analyzing more than 3351 samples, which is experimentally unviable. Therefore, it is imperative to reduce the number of variables before applying the CVA algorithm. The aforementioned variable reduction is achieved using the principal components analysis algorithm (referred to as PCA).

Among all available feature extraction algorithms, PCA is one of the most popular [18]. It is an unsupervised method, which is not based on class tags provided by an expert to guide the classification process. PCA aims at reducing the dimensionality of the problem [19] by condensing the relevant chemometric information contained in the original set of data into a reduced latent variables set [11]. This algorithm transforms the original correlated variables, that is, the reflectances obtained at different wavenumbers, into the principal components (referred to as PCs). The PCs are uncorrelated orthogonal latent variables calculated by the PCA algorithm. PCA extracts hidden and simplified structures within the original data structure explaining the greatest variance [19]. Therefore, this algorithm transforms the original correlated variables into a reduced number of uncorrelated PCs. PCs are calculated from linear combinations of the original variables. They can be understood as the orthogonal directions explaining the higher variance of the data points representing the whole set of samples. PCA calculates the same number of PCs as the number of original variables, although only the PCs explaining the greater amount of variance are retained. To this end, the PCs are ranked from greater to lower variance explained, and only the first PCs explaining a suitable percentage of the overall variance are retained, the others being disregarded. This selection of an adequate number of PCs is done to prevent overfitting [20]. It is crucial to divide the total number of samples into both a calibration and a prediction set.

The mathematical background of the CVA and PCA algorithms can be found in references [16] and [17], respectively.

The classification stage is performed after reducing the dimensionality of the problem, through the use of PCA followed by the CVA algorithm. After applying the PCA+CVA sequence, a classifier algorithm must be used to identify or classify unknown incoming samples according to their pertinence group. To this end, the supervised $k$ nearest neighbor algorithm (referred to as $k \mathrm{NN}$ ) is applied in the classification stage. $k \mathrm{NN}$ is recognized among the most effective and simplest classifiers [14]. The weighted votes or scores are obtained by considering the majority-voting rule of the $k$ nearest neighbors of the calibration sample set. The $k \mathrm{NN}$ algorithm locates the $k$ nearest neighbors in the space defined by the CVs. Then, it assigns $k$ votes to the class of the nearest neighbor, $k-1$ votes to the second nearest neighbor's class and so on until assigning one vote to the $k$-th nearest neighbor's class. Finally, the unknown incoming sample is assigned to the most voted class. Different values of $k$ can be applied, although some authors recommend the use of $k$ values in the range 3-6 [11,21,22]. The $k \mathrm{NN}$ algorithm generates the same number of outputs as classes in the problem, which are often normalized in the $0-1$ interval, denoting the membership level of the incoming samples to each class.

Fig. 1 summarizes the different steps of the process applied in this work to classify unknown incoming samples. 
FTIR spectra

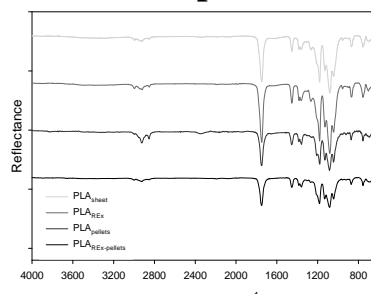

$m \times n$ data matrix

$m$ : number of samples

$n$ : number of wave-numbers

\section{PCA}

Outputs $n$ PCs per sample

$n^{*}<<n$ PCs are retained

\} m \times n ^ { * } \text { data matrix }

\section{CVA}

Outputs $n * *$ CVs per sample

$n^{* *}=$ number classes -1

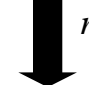

$m \times n^{* *}$ data matrix

$k \mathbf{N N}$ classifier

Outputs clas_numb values $\quad[0,1]$ per sample

Fig. 1. Flowchart of the process applied to classify unknown incoming samples from the FTIR spectral data.

\section{Results and discussion}

A preliminary test was performed in an intent to identify the possible coupling reactions between PLA functional end groups (i.e. $-\mathrm{OH}$ and $-\mathrm{COOH}$ ) and the epoxy groups of the reactive agent using FTIR spectroscopy. A typical IR spectrum for each sample type is shown in Fig. 2. The characteristic peak band assignments for each PLA-based material are summarized in Table 2. 


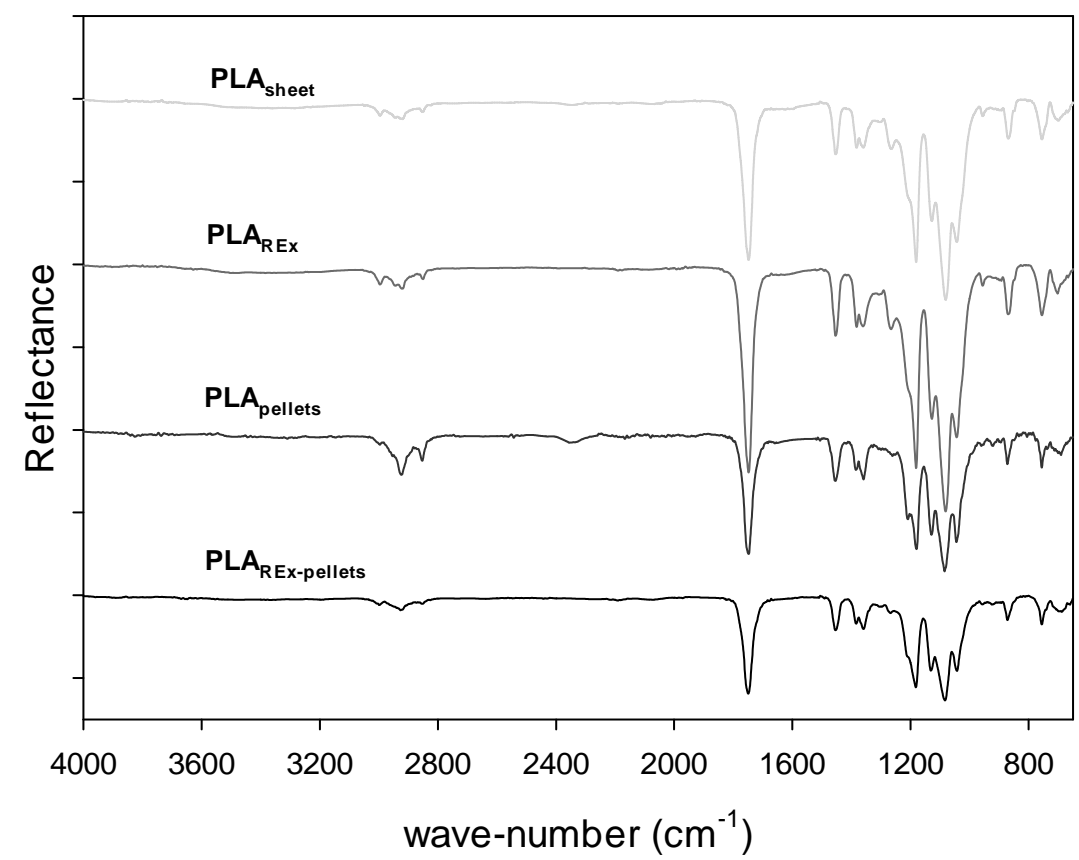

Fig. 2. FTIR reflectance spectra of all investigated samples.

Table 2. Wavenumbers and their corresponding vibrational assignments for PLA-based samples IR spectra $[23,24]$.

\begin{tabular}{ccc}
\hline \hline Wavenumbers, $\boldsymbol{v}\left(\mathbf{c m}^{-1}\right)$ & Assignments & Nature of vibration * \\
\hline 756 & Crystalline phase & - \\
871 & Amorphous phase & - \\
868 & $\mathrm{C}-\mathrm{C}$ & $\mathrm{st}$ \\
1045 & $\mathrm{C}-\mathrm{CH}$ & $\mathrm{st}$ \\
$1093,1130,1184$ & $\mathrm{C}-\mathrm{O}-\mathrm{C}$ & $\mathrm{st}$ as \\
1268 & $\mathrm{C}=\mathrm{O}$ & $\delta$ \\
1360,1382 & $\mathrm{CH}$ & $\delta$ (s and as) \\
1453 & $\mathrm{CH}$ & $\delta$ as \\
1757 & $\mathrm{C}=\mathrm{O}$ & st \\
2880 & $\mathrm{C}-\mathrm{H}$ & st s \\
2945,2994 & $\mathrm{C}-\mathrm{CH}$ & st (s and as) \\
\hline
\end{tabular}

* st: stretching $(v), \delta$ : bending, s: symmetrical, as: asymmetrical

When comparing the FTIR spectra of the polymers studied, before and after modification, no visual differences were observed in the bands corresponding to the functional groups indicated in Table 2. This fact highlights the need to analyze the FTIR spectra by applying suitable mathematical methods, for a proper supervision and control of the PLA modification through the reactive extrusion process.

The data provided by the FTIR instrumental method is used to perform a classification of all investigated PLA-based samples. To this end, the FTIR spectrum obtained for each sample is sequentially analyzed using the PCA and CVA algorithms. Next, the $k \mathrm{NN}$ algorithm is 
applied to classify the unknown incoming samples of the prediction set according to their composition.

The four analyzed groups of samples have different molecular architectures, thus being assumed that this difference is reflected in the FTIR spectra. Therefore, the 79 analyzed samples dealt with, were divided into four groups, that is, 21 were assigned to the PLA $\mathrm{A}_{\text {sheet }}$

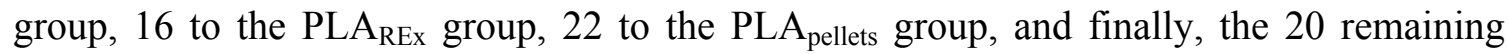
samples were assigned to the PLA $\mathrm{REx}_{\text {-pellets }}$ group. The next step was to randomly divide the four groups into a calibration and a prediction set, which contain approximately $50 \%$ of the PLA samples each. The calibration set includes 40 samples $\left(11\right.$ PLA $_{\text {sheet }}, 8$ PLA REx, 11 PLA $_{\text {pellets }}$ and 10 PLA $_{\text {REx-pellets }}$ samples), whereas the prediction set includes the remaining 39 samples (10 PLA $_{\text {sheet, }} 8$ PLA $_{\text {REx }}, 11$ PLA $_{\text {pellets }}$ and 10 PLA $_{\text {REx-pellets }}$ samples).

The raw spectral data of each sample contains the reflectance value of 3351 wavenumbers within the $650-4000 \mathrm{~cm}^{-1}$ interval. Therefore, the calibration set data matrix contains 40x3351 elements, whereas the matrix describing the prediction set has 39x3351 elements. It is noted that each row of such matrixes correspond to the samples, whereas the different columns in the same row include the spectral information of each sample.

Raw spectral data in the reflectance mode were processed into the first- and secondderivatives, as commonly applied in chemometrics [25]. Results by using the raw spectral data and the first- and second-derivatives of the spectra are presented in next subsections.

\subsection{Raw spectrum data}

This section aims at classifying the samples of the prediction set by using the raw FTIR spectral data. First, the classification model must be calibrated by using the calibration sample set. As previously reported, the spectral data matrix describing the 40 calibration samples includes 40x3351 elements, and therefore the PCA calculates 3351 PCs. To improve the discrimination among groups, once ranked in decreasing order of the explained variance, only the first PCs explaining $99.5 \%$ of the total variance are retained.

Fig. 3 shows the cumulative variance of the first 50 PCs arising from the PCA algorithm. It is shown that by only retaining the 18 first PCs, $99.5 \%$ of the total variance is explained, thus greatly reducing the dimensionality of the problem. This facilitates the subsequent application of the CVA algorithm. 


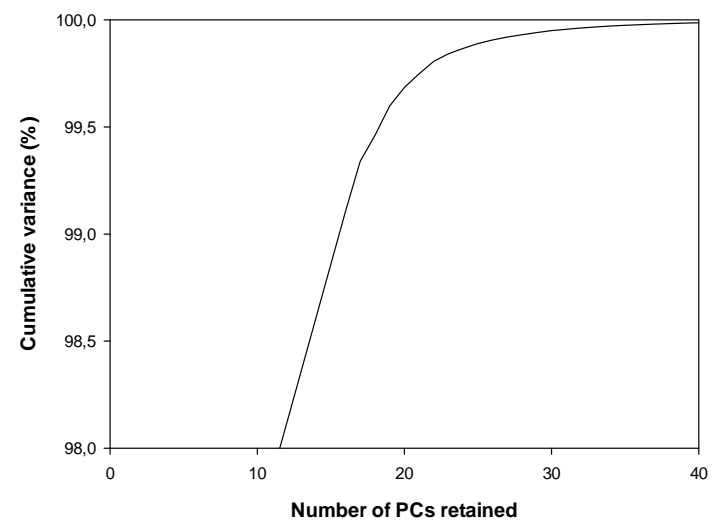

Fig. 3. Cumulative variance versus the number of PCs retained for the calibration set.

Fig. 4 summarizes the results attained based on the PCA + CVA approach, when dealing with 40 calibration samples and 39 prediction samples.

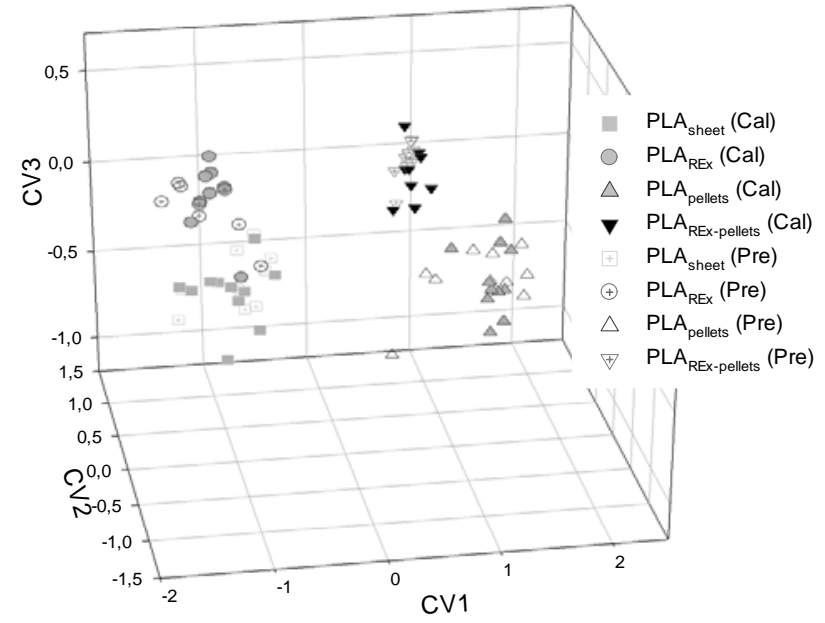

Fig. 4. Raw spectral data. Plot of the calibration and prediction samples in the space defined by the three CVs arising from the CVA previous application of the PCA algorithm and retaining the first 18 PCs.

After application of the $k \mathrm{NN}$ classifier, the results of classification according to the PCA (18 $\mathrm{PCs})+\mathrm{CVA}+k \mathrm{NN}$ approach are 36 correctly classified samples $(k=3,4,5,6)$ out of 39 prediction samples $(92.31 \%$ classification rate).

\subsection{First derivative of the spectrum}

This section classifies the samples of the prediction from the first derivative of the FTIR spectra. The first derivative of the spectral information is carried out by means of the Savitzky-Golay algorithm, taking into account five left-sided and five right-sided spectral points. By deriving the 40x3351 spectral data matrix of the 40 calibration samples, it results in a 40x3341 data matrix, which is the input of the PCA algorithm. Therefore, the PCA 
algorithm calculates $3341 \mathrm{PCs}$, the first 19 of them explaining $99.5 \%$ of the total variance, as shown in Fig. 5.

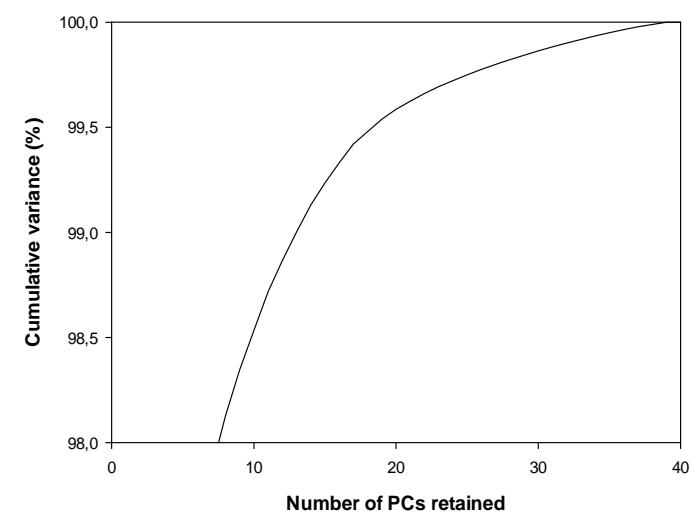

Fig. 5. Calibration set in first derivative mode of the spectral data. Cumulative variance versus the number of PCs retained.

Fig. 6 shows the results attained based on the PCA + CVA approach when dealing with 40 calibration samples and 39 prediction samples.

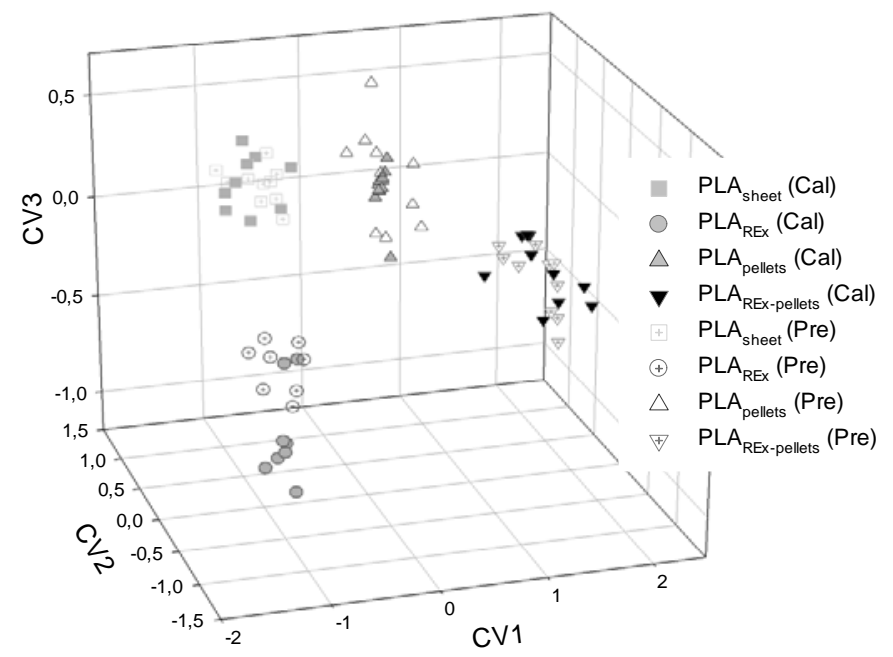

Fig. 6. First derivative mode of the FTIR spectral data. Plot of the calibration and prediction samples in the space defined by the three CVs arising from the CVA previous application of the PCA algorithm and retaining the first 19 PCs.

Classification results attained by the $k \mathrm{NN}$ algorithm, after the PCA (19 PCs) + CVA dimensionality reduction stage are 39 correctly classified samples $(k=3,4,5,6)$ out of 39 prediction samples (100\% classification rate).

\subsection{Second derivative of the spectrum}

Finally, the second derivative of the raw spectral data is calculated by applying the SavitzkyGolay algorithm, taking into account 10 left-sided and 10 right-sided spectral points. The 
second derivative matrix of the 40 calibration samples has $40 \times 3331$ elements. This matrix is the input of the PCA algorithm, which computes 3331 PCs, the first 12 of them explaining $99.5 \%$ of the total variance, as shown in Fig. 7.

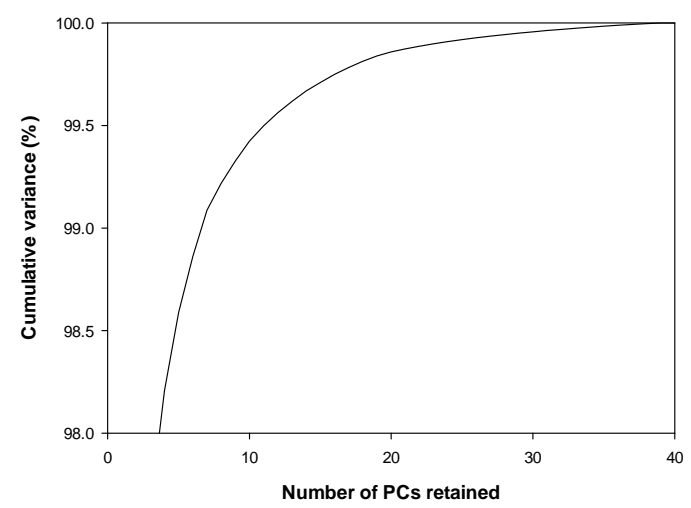

Fig. 7. Calibration set in second derivative mode of the spectral data. Cumulative variance versus the number of PCs retained.

Fig. 8 shows the results attained based on the PCA + CVA approach when analyzing the second derivative of the spectral data.

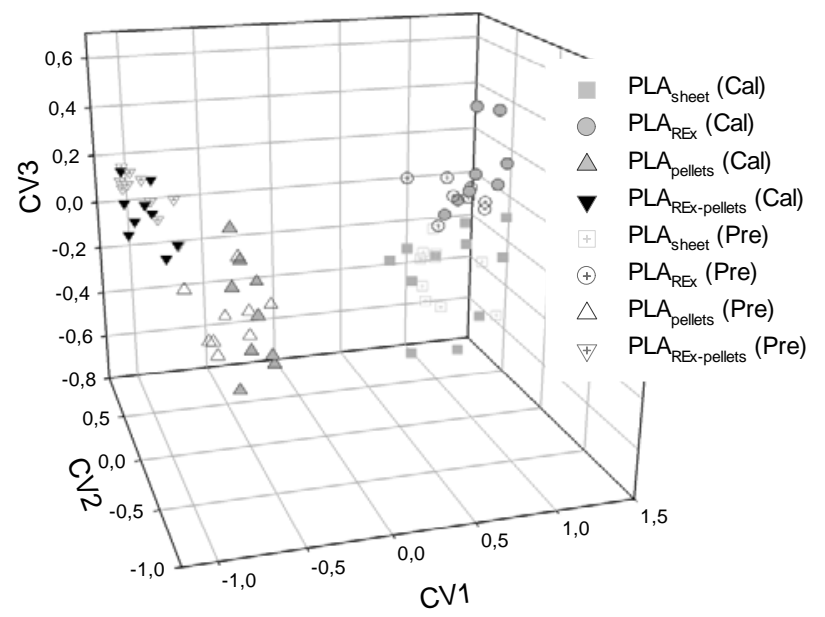

Fig. 8. Second derivative mode of the FTIR spectral data. Plot of the calibration and prediction samples in the space defined by the three CVs arising from the CVA previous application of the PCA algorithm and retaining the first 12 PCs.

Classification results attained through application of the $k \mathrm{NN}$ algorithm, after the PCA (12 PCs) + CVA dimensionality reduction stage are 36,37, 38 and 39 correctly classified samples ( $k=3,4,5$ and 6 , respectively) out of 39 prediction samples $(92.31 \%, 94.87 \%, 97.44 \%$ and $100 \%$ classification rate, respectively). 


\subsection{Summary of results}

As explained, the $k \mathrm{NN}$ classifier algorithm produces four output values for each incoming sample, that is, one output per class, whose values are within the interval $[0,1]$. Each output value quantifies the membership level of the sample under analysis to each class. Output values above 0.5 express pertinence to the considered class. However, output membership values below or equal to this threshold value indicate that the sample does not belong to the considered class. It is noted that a unity membership level indicates that the sample is classified in a determined class with total certainty, whereas a membership level of 0.55 has high uncertainty. The PRESS (predictive residual error sum of squares) indicator is used to assess the accuracy of the classification results. It is calculated as,

$$
\operatorname{PRESS}=\sum_{i=1}^{n}\left(y_{i}-\hat{y}_{i}\right)^{2}
$$

where $y_{i}$ is the output value of the $k \mathrm{NN}$ algorithm within the range $[0,1]$ for the $i$-th prediction sample and $\hat{y}_{i}$ is the real value of $y_{i}$, being either 0 or 1 . It is noted that the values of $\hat{y}_{i}$ (either 1 or 0 ) are known since they are settled by an expert. The accuracy of the output values of the $k \mathrm{NN}$ classifier increases when decreasing the values of the PRESS.

Table 3 summarizes the classification results of the prediction set samples attained in this paper when using the raw spectral data, and the first and second derivatives of the spectra.

Table 3. Results summary when applying the PCA $+\mathrm{CVA}+k \mathrm{NN}$ approach

\begin{tabular}{cccc}
\hline Type of data & $\begin{array}{c}\boldsymbol{k} \mathbf{N N} \\
\text { classifier }\end{array}$ & $\begin{array}{c}\text { Success rate of } \\
\text { classification }\end{array}$ & PRESS \\
\hline \multirow{2}{*}{ Raw spectral } & $k=3$ & $36 / 39(92.3 \%)$ & 3.61 \\
data & $k=4$ & $36 / 39(92.3 \%)$ & 3.54 \\
& $k=5$ & $36 / 39(92.3 \%)$ & 3.66 \\
$k=6$ & $36 / 39(92.3 \%)$ & 3.86 \\
\hline \multirow{2}{*}{ First derivative of } & $k=3$ & $39 / 39(100 \%)$ & 0.22 \\
spectra & $k=4$ & $39 / 39(100 \%)$ & 0.20 \\
& $k=5$ & $39 / 39(100 \%)$ & 0.18 \\
First derivative of & $k=6$ & $39 / 39(100 \%)$ & 0.19 \\
spectra & $k=4$ & $36 / 39(92.3 \%)$ & 2.44 \\
& $k=5$ & $37 / 39(94.9 \%)$ & 2.22 \\
& $k=6$ & $38 / 39(97.4 \%)$ & 2.21 \\
& & & 2.33 \\
\hline
\end{tabular}

From Table 3 it is deduced that the best results are attained when dealing with the first derivative of the spectral data, although when dealing with both the raw spectral data and the second derivative of the spectra the success rate in identifying incoming samples not included in the calibration set is always higher than $92 \%$. 


\section{Conclusions}

This paper has proposed a very fast, noninvasive, accurate and easy-to-apply method to detect structural changes of PLA samples modified through reactive extrusion. The proposed method does not require any prior sample treatment, and being compatible for continuous control of the production process of the PLA. For this purpose, spectral data obtained from FTIR spectroscopy has been analyzed in combination with multivariate feature extraction methods such as PCA, PCA + CVA and the kNN classifier.

The experimental results summarized in this paper have proved that the proposed approach allows achieving high classification rates, always beyond $92 \%$ and up to $100 \%$ when dealing with the first derivative of the spectral data. The obtained reliable and accurate results show the potential of the proposed method to supervise and control the topological modification of PLA through reactive extrusion in pre-industrial setting.

\section{Acknowledgements}

The authors acknowledge the financial support from the Spanish Ministry of Economy and Competitiveness through the Project MAT2016-80045-R (AEI/FEDER,UE).

\section{References}

[1] J.-M. Raquez, R. Narayan, P. Dubois, Recent Advances in Reactive Extrusion Processing of Biodegradable Polymer-Based Compositions, Macromol. Mater. Eng. 293 (2008) 447-470. doi:10.1002/mame.200700395.

[2] J. Cailloux, O.O. Santana, E. Franco-Urquiza, J.J. Bou, F. Carrasco, M.L. Maspoch, Sheets of branched poly(lactic acid) obtained by one-step reactive extrusion-calendering process: physical aging and fracture behavior, J. Mater. Sci. 49 (2014) 4093-4107. doi:10.1007/s10853-014-8101-y.

[3] Y.-M. Corre, J. Duchet, J. Reignier, A. Maazouz, Melt strengthening of poly (lactic acid) through reactive extrusion with epoxy-functionalized chains, Rheol. Acta. 50 (2011) 613-629. doi:10.1007/s00397-011-0538-1.

[4] Y. Di, S. Iannace, E. Di Maio, L. Nicolais, Reactively Modified Poly(lactic acid): Properties and Foam Processing, Macromol. Mater. Eng. 290 (2005) 1083-1090. doi:10.1002/mame.200500115.

[5] J. Liu, L. Lou, W. Yu, R. Liao, R. Li, C. Zhou, Long chain branching polylactide: Structures and properties, Polymer (Guildf). $51 \quad$ (2010) 5186-5197. doi:10.1016/j.polymer.2010.09.002.

[6] S. Yang, Z.-H. Wu, W. Yang, M.-B. Yang, Thermal and mechanical properties of chemical crosslinked polylactide (PLA), Polym. Test. 27 (2008) 957-963. doi:10.1016/j.polymertesting.2008.08.009. 
[7] J. Cailloux, O.O. Santana, M.L. Maspoch, J.J. Bou, F. Carrasco, Using viscoelastic properties to quantitatively estimate the amount of modified poly(lactic acid) chains through reactive extrusion, J. Rheol. (N. Y. N. Y). 59 (2015) 1191-1227. doi:10.1122/1.4928071.

[8] B.J. Crosby, M. Mangnus, W. de Groot, R. Daniels, T.C.B. McLeish, Characterization of long chain branching: Dilution rheology of industrial polyethylenes, J. Rheol. (N. Y. N. Y). 46 (2002) 401-426. doi:10.1122/1.1451083.

[9] T. Malmgren, J. Mays, M. Pyda, Characterization of poly(lactic acid) by size exclusion chromatography, differential refractometry, light scattering and thermal analysis, J. Therm. Anal. Calorim. 83 (2006) 35-40. doi:10.1007/s10973-005-7066-0.

[10]L.E. Rodriguez-Saona, M.M. Giusti, M. Shotts, 4 - Advances in Infrared Spectroscopy for Food Authenticity Testing, in: Adv. Food Authent. Test., 2016: pp. 71-116. doi:10.1016/B978-0-08-100220-9.00004-7.

[11]J.-R. Riba Ruiz, T. Canals, R. Cantero, Supervision of Ethylene Propylene Diene MClass (EPDM) Rubber Vulcanization and Recovery Processes Using Attenuated Total Reflection Fourier Transform Infrared (ATR FT-IR) Spectroscopy and Multivariate Analysis, Appl. Spectrosc. 71 (2017) 141-151. doi:10.1177/0003702816653131.

[12] J.-R.J.-R. Riba, T. Canals, R. Cantero, H. Iturriaga, Potential of infrared spectroscopy in combination with extended canonical variate analysis for identifying different paper types, Meas. Sci. Technol. 22 (2011) 25601. doi:10.1088/0957-0233/22/2/025601.

[13] J.-R.J.-R. Riba, T. Canals, R. Cantero, Recovered Paperboard Samples Identification by Means of Mid-Infrared Sensors, IEEE Sens. J. 13 (2013) 2763-2770. doi:10.1109/JSEN.2013.2257943.

[14] J.-R.J.-R. Riba Ruiz, T. Canals, R. Cantero Gomez, T.C. Parelló, R. Cantero Gómez, Comparative Study of Multivariate Methods to Identify Paper Finishes Using Infrared Spectroscopy, IEEE Trans. Instrum. Meas. 61 (2012) 1029-1036. doi:10.1109/TIM.2011.2173048.

[15]J. Pan, K.L. Nguyen, Development of the Photoacoustic Rapid-Scan FT-IR-Based Method for Measurement of Ink Concentration on Printed Paper, Anal. Chem. 79 (2007) 2259-2265. doi:10.1021/AC061732Y.

[16]L. Nørgaard, R. Bro, F. Westad, S.B. Engelsen, A modification of canonical variates analysis to handle highly collinear multivariate data, J. Chemom. 20 (2006) 425-435. doi:10.1002/cem.1017.

[17]R. A. Johnson, D.W. Wichern, Applied Multivariate Statistical Analysis, 6th editio, Prentice-Hall, Englewood Cliffs, NJ (USA), 2007.

[18]W. Lai, X. Zeng, J. He, Y. Deng, Aesthetic defect characterization of a polymeric polarizer via structured light illumination, Polym. Test. 53 (2016) 51-57. doi:10.1016/j.polymertesting.2016.05.011.

[19]D. Song, J. Gao, X. Li, L. Lu, Evaluation of aging behavior of polypropylene in natural environment by principal component analysis, Polym. Test. 33 (2014) 131-137. 
doi:10.1016/j.polymertesting.2013.11.014.

[20]N. Bhattacharyya, R. Bandyopadhyay, M. Bhuyan, B. Tudu, D. Ghosh, A. Jana, Electronic Nose for Black Tea Classification and Correlation of Measurements With "Tea Taster" Marks, IEEE Trans. Instrum. Meas. 57 (2008) 1313-1321. doi:10.1109/TIM.2008.917189.

[21]L.A. Berrueta, R.M. Alonso-Salces, K. Héberger, Supervised pattern recognition in food analysis, J. Chromatogr. A. 1158 (2007) 196-214. doi:10.1016/j.chroma.2007.05.024.

[22] J.-R. Riba Ruiz, T. Canals Parello, R. Cantero Gomez, Identification of NR and EPDM Samples by Means of Thermogravimetric Analysis and Multivariate Methods, IEEE Sens. J. 16 (2016) 7705-7712. doi:10.1109/JSEN.2016.2603172.

[23]D. Garlotta, A Literature Review of Poly(Lactic Acid), J. Polym. Environ. 9 (2001) 6384. doi:10.1023/A:1020200822435.

[24] C.M.B. Gonçalves, J.A.P. Coutinho, I.M. Marrucho, Optical Properties, in: Poly(Lactic Acid), John Wiley \& Sons, Inc., Hoboken, NJ, USA, 2010: pp. 97-112. doi:10.1002/9780470649848.ch8.

[25] T. Canals, J.R. Riba, R. Cantero, J. Cansino, D. Domingo, H. Iturriaga, Characterization of paper finishes by use of infrared spectroscopy in combination with canonical variate analysis, Talanta. 77 (2008) 751-757. doi:10.1016/j.talanta.2008.07.059. 